\title{
A Three-Dimensional Motion Anlaysis of Horse Rider in Wireless Sensor Network Environments
}

\author{
Jae-Neung Lee \\ Dept. of Control and Instrumentation Engineering, \\ Chosun University, 375 Seosuk-dong \\ Gwangju, Korea
}

\author{
Keun-Chang Kwak* \\ Dept. of Control and Instrumentation Engineering, \\ Chosun University, 375 Seosuk-dong \\ Gwangju, Korea
}

\begin{abstract}
This paper constructed a database of the national representative level of a professional horse-rider by wearing a motion-capture suit attached with 16 inertial sensors under an inertial sensor-based wireless network environment, then made a visual comparative analysis through a few methods (graphical and statistical) on the values of all motion features (elbow angle, knee angle, knee-elbow distance, backbone angle and hip position) classified depending on horse types (using two horses named Warm-blood and Thoroughbred) and footpace types (at a trot and a canter) and obtained by various methods of calculating Euclidean distance, the second cosine, maximum and minimum values, and made a comparative analysis depending on motion features of a horse-rider by using MVN studio software. In the study, the experimental results confirmed the validity of the proposed method of obtaining the motion feature database of a horse-rider in the wireless sensor network environment and making an analytical system.
\end{abstract}

Keywords-3D motion capture and analysis; inertial sensor; wireless network

\section{INTRODUCTION}

A lot of people are doing exercises to keep a good body shape. Especially, everybody knows that obesity is good neither for appearance nor for health. Horse-riding is a good sport of keeping good health and body line. It is possible to analyze and properly coach the postures of a horse-rider by the analysis of the so-called horse-riding motions under the wireless sensor network environment. Particularly, horseriding is an exercise with a special trait that a horse-rider and a horse alive should be joined together. It can be helpful for a horse-rider to build up physical health and spiritual growth. In addition, the horse-riding is a physical exercise of the whole body helpful to improve body's balance and flexibility for general physical developments, and it is also a spiritual exercise helpful to bring a spirit of boldness and sound thinking and to cultivate humanity through the learning process of being kind to animals. In summary of the horseriding related mental, physical and psychological effects, it helps mentally to improve self-confidence through the talks with animals in love, learn social order in the process of horseriding activities and cultivate patience. In addition, as it is an exercise of the whole body, it helps physically to improve blood circulation by using muscles and joints and general body adaptability and flexibility in relation to functional recovery, a sense of balance and a change in speed by stimulating all the nerves of several body parts. Furthermore, it has positive psychological impacts of having a satisfactory feeling about properly dealing with a big animal like a horse, a respectful mind for the dignity of life and an emotional growth in inter-personal relationships through enhancement of active attitudes in general. Finally, it is directly effective to correct body postures, enhance bowel function, enlarge lung capacity, prevent arthritis, anemia and constipation, build up courage, increase back flexibility, promote body rhythms and strength pelvis [1-3].

However, the horse-riding is not effective if correct body postures are not learned and maintained properly in the course of the exercise. Therefore, in order to make the most effective achievement within the shortest period of time, it is necessary to get a coaching session to check what is wrong with the motions of a horse-rider. Recently a lot of studies have been made by using inertial sensors at the wireless sensor network environment to carry out the most effective coaching session of the horse-riding sport. Luinge [4] proposed a method of accurately measuring the size of a person by using inertial sensors and angular velocity sensors.

Zhou [5] suggested a new human motion tracking system using two wearable inertial sensors that are placed near the wrist and elbow joints of the upper limb. Lee[6] suggested Sensor fusion and calibration for motion captures using accelerometers. Zhu [7] presented a real time motion-tracking system using tri-axis micro electromechanical accelerometers. Cheng [8] suggested the results of a set of network traffic experiments that were designed to investigate the suitability of conventional wireless motion sensing system design which generally assumes in-network processing - as an efficient and scalable design for use in sports training. Venkatraman [9] analyzed a behavior of the animal is further extracted from the recorded acceleration data using neural network based pattern recognition algorithms. Ghasemzadeh [10] suggested a golf swing training system which incorporates wearable motion sensors to obtain inertial information and provide feedback on the quality of movements. Kevin [11] analyzed a theory, design, and evaluation of a miniature, wireless IMU (Initial Measurement Unit) that precisely measures the dynamics of a golf club used in putting. Mariani [12] described 3D gait assessment in young and elderly subjects using foot-worn inertial sensors. Yujin[13] suggested Upper Body Motion Tracking With Inertial Sensors. Lijun [14] analyzed A Practical Calibration Method on MEMS Gyroscope. Wei [15] suggested calibration of low-precision MEMS inertial sensor. Cao [16] performed 3D dynamics analysis of a golf full swing by fusion inertial sensors and vision data. Jung [17] analyzed 
smart shoes. Chan [18] proposed a virtual reality dance training system using motion capture technology. Frosio [19] analyzed automatic calibration of MEMS accelerometers.

However, a variety of inertial sensor-based studies have been made so far, but no study has been made about an analysis on the motions of a horse-rider in a wireless network environment. Thus, in this paper, a database is constructed by collecting the motions of a professional horse-rider wearing a motion-capture suit under the inertial sensor-based wireless network environment. At this time, two representative types of horses, named Warm-blood and Thoroughbred, were selected for this study. Actual horse-riding sessions were made to analyze the postures of a professional horse-rider by measuring and calculating all the motion feature values of elbow angle, knee angle, knee-elbow distance, backbone angle and hip $y$-axis position at the two representative horse-riding footpace types, rising trot and canter.

The results of the experiments made at MVN Studio with MATLAB confirmed the validity of the method of analyzing the motions of a horse-rider in a wireless sensor network environment suggested in this study, in which all the motion data of a horse-rider were collected in the wireless sensor network environment [20] to make a comparative analysis on all the horse-riding postures carefully.

\section{METHOD OF CONSTRUCTING AN INERTIAL SENSOR-}

\section{BASED WIRELESS NETWORK ENVIRONMENT AND A DATABASE}

This chapter describes a method of constructing an inertial sensor-based wireless network environment and a motion database. All the motion data of a horse-rider are received to a computer through the MVN motion capture system constructed with inertial sensors made by Xsens Co. Then, all the data are compared by using respective calculation methods. Figure 2 below illustrated the steps of collecting and constructing a database of motions in a wireless sensor network environment. Differently from an optical sensorbased motion capture system, the MVN motion capture system can capture the entire body motions wirelessly without using a camera. In addition, the MVN motion capture system is portable for convenient indoor-outdoor uses.

Figure 1 illustrates a process of wireless sensor network made by Xsens Co. The MTX sensor used for constructing the database is a small and light 3DOF Human orientation tracker which provides drift-free kinematic data, precise three-axis acceleration, three-axis gyroscope and three-axis geomagnetic values. Figure 3 shows the sensor used for constructing a database.

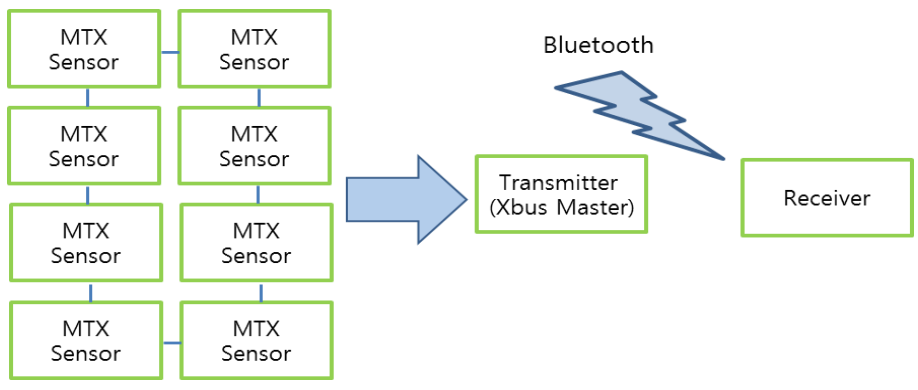

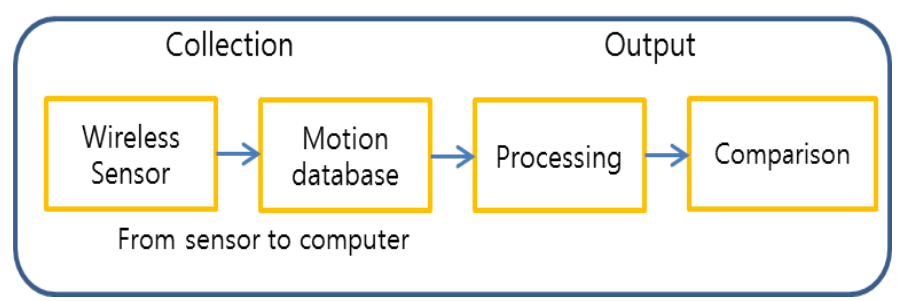

Fig. 2. Data collection and output processing
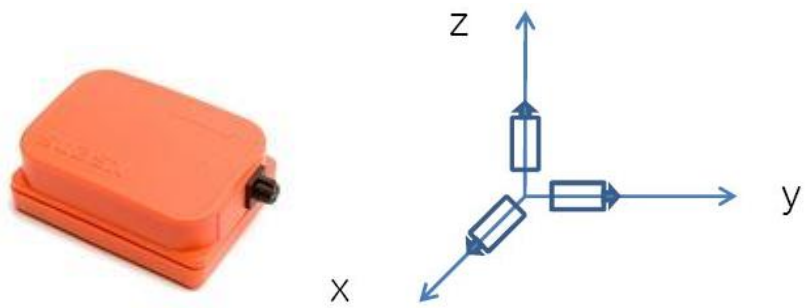

Fig. 3. MTX sensor: 3DOF human orientation tracker

In order to collect data, this study used a subject, a national representative level of a professional horse-rider whose height is $164 \mathrm{~cm}$ with her foot size of $235 \mathrm{~mm}$. She worn the MVN, the inertial sensor-based 3D motion capture suit made by Xsens, to collect data, subsequently riding on the horses, thoroughbred whose height is $160 \mathrm{~cm}$ and Warm-blood whose height is $150 \sim 173 \mathrm{~cm}$. The period of time taken for measurement of one file was about 1 2 minutes and 15 data were collected depending on footpace types. There are 4 horse footpace types such as walk, trot, canter and gallop. A horse usually goes as far as $130 \mathrm{~m}$ for a minute, approximately $8 \mathrm{~km}$ for an hour at a walk. It usually moves as far as $220 \mathrm{~m}$ for a minute, approximately $13 \mathrm{~km}$ for an hour at a rising trot, one specific type of trots. It generally moves as far as $350 \mathrm{~m}$ for a minute, approximately $21 \mathrm{~km}$ for an hour at a canter. It moves as far as $1000 \mathrm{~m}$ for a minute, approximately $60 \mathrm{~km}$ or even $72 \mathrm{~km}$ for an hour at a gallop. The horses used in the experiments of this study were made to move at the two footpace types, a rising trot and a canter. The measurement frame rate was 100 frames per second. Figure 4 (a) show the two horse types respectively, Warm-blood and Thoroughbred, on which the horse-rider was sitting, in the process of constructing the actual DB. Figure 4 (b) illustrates all the 16 body parts attached with inertial sensors, differently marked for her visible front and lateral sides and her invisible back side.

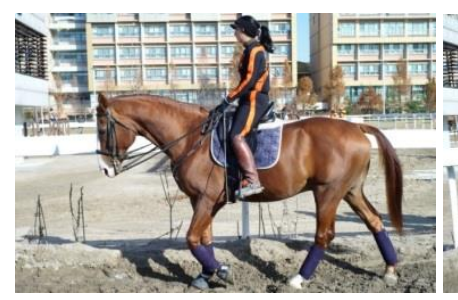

(a)

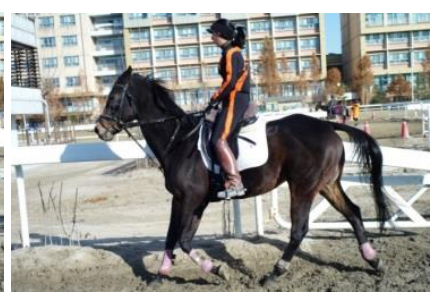

(b)
Fig. 4. (a) Warm-blood of Actual horse-rider's postures (b) Thoroughbred of Actual horse-rider's postures

Fig. 1. Process of wireless sensor network 


\section{METHOD OF EXTRACTING FEATURES FROM MOTIONS OF HORSE-RIDER}

If body postures are not properly learned and maintained by a horse-rider, the horse-riding exercise may bring not a positive, but a negative effect. Therefore, it is necessary to make an analysis on the horse-riding postures to clearly check what is wrong with the motions of the horse-rider. The following method is presented to make a comparative analysis on 5 motion features (elbow angle, knee angle, elbow-knee distance, backbone angle, hip position).

\section{A. Elbow Angle}

The values of motion features collected by the sensors attached at body parts, $\mathrm{A}$ (wrist), B(elbow) and $\mathrm{C}$ (shoulder), are extracted from the $\mathrm{DB}$ to define three coordinates, $\mathrm{A}, \mathrm{B}$ and $\mathrm{C}$ through Eq. (1). Figure 5 (a) shows MVN studio motion capture software. Figure 5 (b) illustrates a method of obtaining an elbow angle.

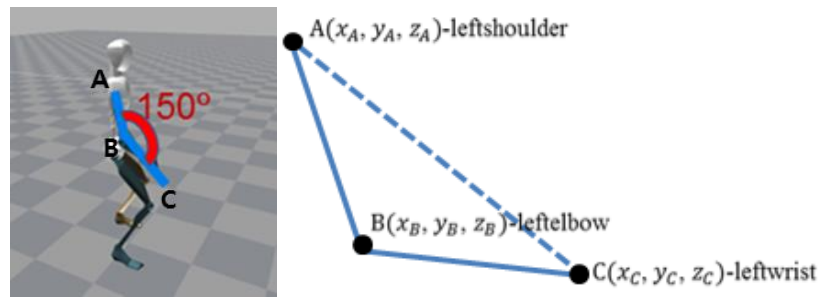

(a)

(b)

Fig. 5. (a) MVN studio software (b) Method of obtaining an elbow angle

$\mathrm{A}=x_{A}, y_{A}, z_{A}, \mathrm{~B}=x_{B}, y_{B}, z_{B}, \mathrm{C}=x_{C}, y_{C}, z_{C}$

Besides, all the respective distances among feature positions, $\mathrm{A}$ (wrist), $\mathrm{B}$ (elbow), $\mathrm{C}$ (shoulder), are obtained in Euclidean geometry through Eq.(2)

$$
\begin{aligned}
& \overline{A B}=\sqrt{\left.\left(x_{A}-x_{B}\right)^{2}+\left(y_{A}-y_{B}\right)^{2}+\left(z_{A}-z_{B}\right)^{2}\right)}=c \\
& \overline{B C}=\sqrt{\left.\left(x_{B}-x_{C}\right)^{2}+\left(y_{B}-y_{C}\right)^{2}+\left(z_{B}-z_{C}\right)^{2}\right)}=a \\
& \overline{C A}=\sqrt{\left.\left(x_{C}-x_{A}\right)^{2}+\left(y_{C}-y_{A}\right)^{2}+\left(z_{C}-z_{A}\right)^{2}\right)}=b
\end{aligned}
$$

The respective distances among the feature positions are obtained and applied to the following Eq.(3).

$$
\begin{aligned}
& a^{2}=b^{2}+c^{2}-2 b c \cos A \\
& b^{2}=c^{2}+a^{2}-2 c a \cos B \\
& c^{2}=a^{2}+b^{2}-2 a b \cos C
\end{aligned}
$$
angle.

A change is made into Eq.(4), so as to calculate an elbow

$$
\text { elbow angle }=\cos ^{-1}\left(\frac{c^{2}+a^{2}-b^{2}}{2 c a}\right)
$$

\section{B. Knee Angle}

The values of motion features collected by the sensors attached at body parts, A(left hip), B(left knee) and C(left ankle), are extracted from the DB to define three coordinates,
$\mathrm{A}, \mathrm{B}$ and $\mathrm{C}$ in the same way shown in case of the elbow angle. The distances among feature positions, A(left hip), B(left knee), C(left ankle), are obtained in Euclidean geometry. The respective distances among the feature positions are obtained and then applied to Eq. (3). A change is made into Eq. (5), so as to calculate the knee angle.

$$
\text { knee angle }=\cos ^{-1}\left(\frac{c^{2}+a^{2}-b^{2}}{2 c a}\right)
$$

Figure 6 (a) shows MVN studio motion capture software. Figure 6 (b) illustrates a method of obtaining a knee angle.

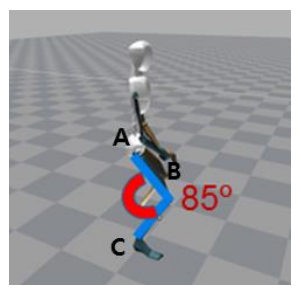

(a)

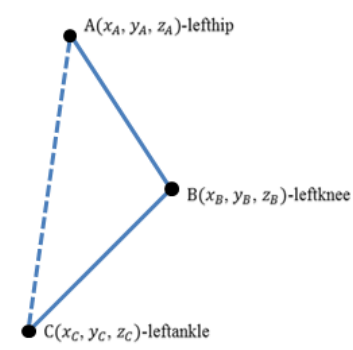

(b)
Fig. 6. (a) MVN studio software (b) Method of obtaining a knee angle

\section{Elbow-Knee Distance}

The values of motion features collected by the sensors attached at body parts, $\mathrm{A}$ (left elbow) and B(right elbow), are extracted from the DB to define two coordinates, A and B as shown in Figure 6 (b). An elbow distance between the features $\mathrm{A}$ and $\mathrm{B}$ is obtained through Eq. (6).

$$
\text { Elbow distance }=\sqrt{\left.\left(x_{A}-x_{B}\right)^{2}+\left(y_{A}-y_{B}\right)^{2}+\left(z_{A}-z_{B}\right)^{2}\right)}
$$

In the same way described above, the values of body features collected by the sensors attached at body parts, C(left knee) and $\mathrm{D}$ (right knee), are extracted from the DB to define two coordinates, $\mathrm{C}$ and $\mathrm{D}$ as shown in Figure 7. A knee distance between the features $\mathrm{C}$ and $\mathrm{D}$ is obtained through Eq. (7)

$$
\text { knee distance }=\sqrt{\left.\left(x_{A}-x_{B}\right)^{2}+\left(y_{A}-y_{B}\right)^{2}+\left(z_{A}-z_{B}\right)^{2}\right)}
$$

Figure 7 illustrate methods of obtaining an elbow distance and a knee distance, respectively.

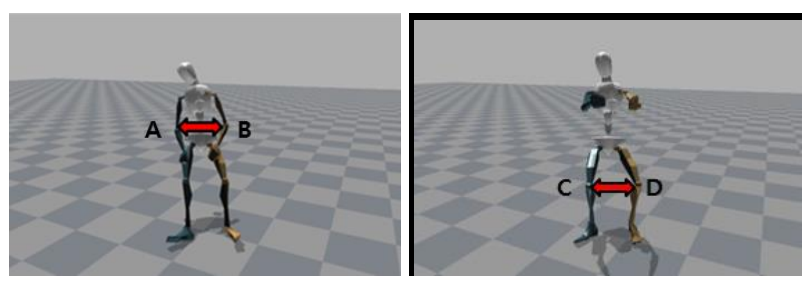

Fig. 7. Method of obtaining an elbow and knee distance

\section{Backbone Angle}

The values of motion features collected by the sensors attached at body parts, $\mathrm{A}$ (neck), $\mathrm{B}$ (chest) and $\mathrm{C}$ (chest), are extracted from the DB to define three coordinates, A, B and C 
in the same way shown above in case of the elbow angle. The distances among feature positions, A(neck), B(chest 3 ) and $\mathrm{C}$ (chest), are obtained in Euclidean geometry. The respective distances among the feature positions are obtained and then applied to Eq. (3). A change is made into Eq. (8), so as to calculate a backbone angle.

$$
\text { backbone angle }=\cos ^{-1}\left(\frac{c^{2}+a^{2}-b^{2}}{2 c a}\right)
$$

Figure 8 (a) shows MVN studio motion capture software. Figure 8 (b) illustrates a method of obtaining a backbone angle.

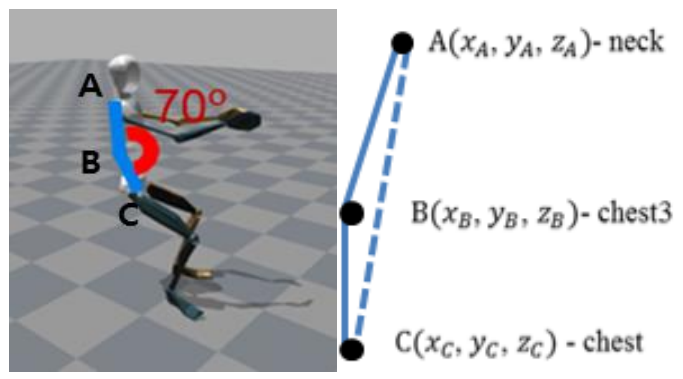

(a)

(b)

Fig. 8. (a) MVN studio software (b) Method of obtaining a backbone angle

\section{E. Hip Position}

The value of a body feature collected by the sensor attached at a body part, A(hip), is extracted from the DB to define a coordinate, $\mathrm{A}(\mathrm{x}, \mathrm{y}, \mathrm{z})$. As the hip position is centered at horse-riding, the movement of $\mathrm{A}(\mathrm{y})$ axis is in use.

$$
\text { Hip Position }=\mathrm{A}\left(Y_{A}\right)
$$

\section{EXPERIMENTAL RESULTS}

In the experiments of this study, data were collected by making two horse types (Warm-blood and Thoroughbred) at two footpace types (rising trot and canter) 15 times, so that 4 cycles of data were extracted for comparative analysis. It was confirmed that there was differences in horse-rider's postures through different values of respective angles and distances. The right elbow and knee are marked in red dotted lines.

\section{A. Warm-blood at a Trot}

The following figure shows that repeating 200 frames were extracted out of approximately 10000 frames to demonstrate 4 cycles (2.5 seconds). As shown in the Figure below, the elbow and knee angles remain at the range of about 130 150 degrees and about 130-170 degrees, respectively. The elbow and knee distances stay at the range of $18 \sim 27 \mathrm{~cm}$ and $15 \sim 18 \mathrm{~cm}$, respectively. The backbone angle remains at the range of 170 177 degrees. The Hip positions moves within the range of $30 \sim 37 \mathrm{~cm}$. Figure 9 shows the average feature values of 15 data.
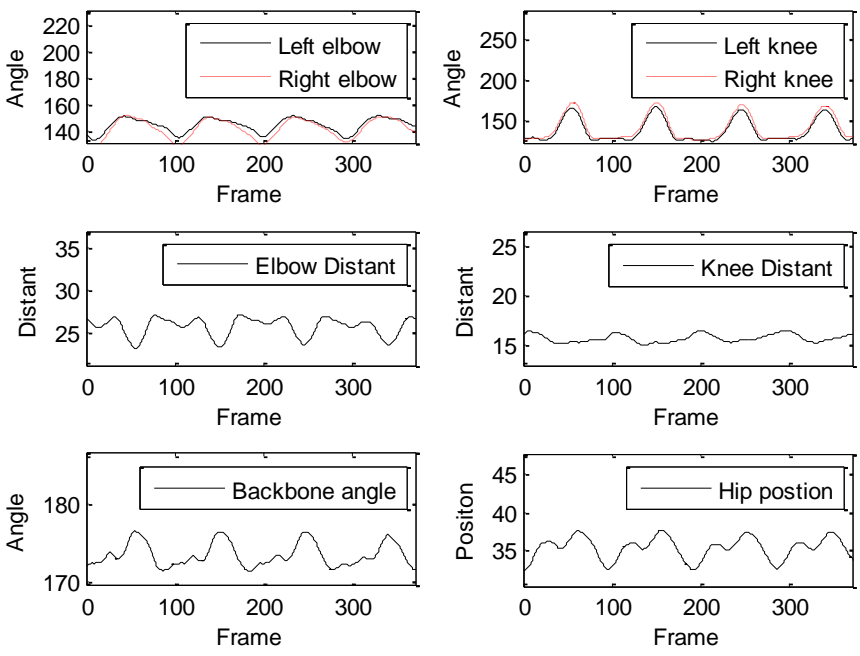

Fig. 9. Feature values of Warm-blood at a trot

\section{B. Warm-blood at a Canter}

Figure 10 shows that repeating 300 frames were extracted out of approximately 10000 frames to demonstrate 4 cycles (3 seconds). As shown in the Figure below, the elbow and knee angles remain at the range of about 130 160 degrees and 130 140 degrees, respectively. The elbow and knee distances stay at the range of $25 \sim 29 \mathrm{~cm}$ and $15 \sim 18 \mathrm{~cm}$, respectively. The backbone angle remains at the range of 170 177 degrees. The Hip positions moves within the range of $30 \sim 37 \mathrm{~cm}$. Figure 10 shows the average feature values of 15 data.
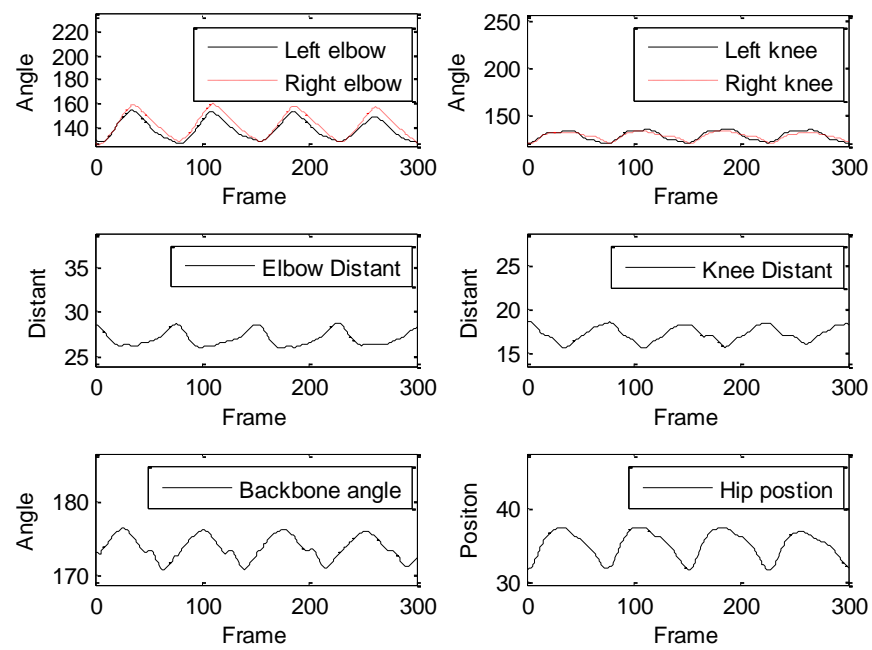

Fig. 10. Feature values of Warm-blood at a canter

\section{Thoroughbred at a Trot}

Figure 11 shows that repeating 200 frames were extracted out of approximately 10000 frames to demonstrate 4 cycles (2.5 seconds). As shown in the Figure below, the elbow and knee angles remain at the range of about 135 160 degrees and about 120 160 degrees, respectively. 
The elbow and knee distances stay at the range of $22 \sim 25 \mathrm{~cm}$ and $14 \sim 16 \mathrm{~cm}$, respectively. The backbone angle remains at the range of 170 177 degrees. The Hip positions moves within the range of $34 \sim 40 \mathrm{~cm}$. Figure 11 shows the average feature values of 15 data.
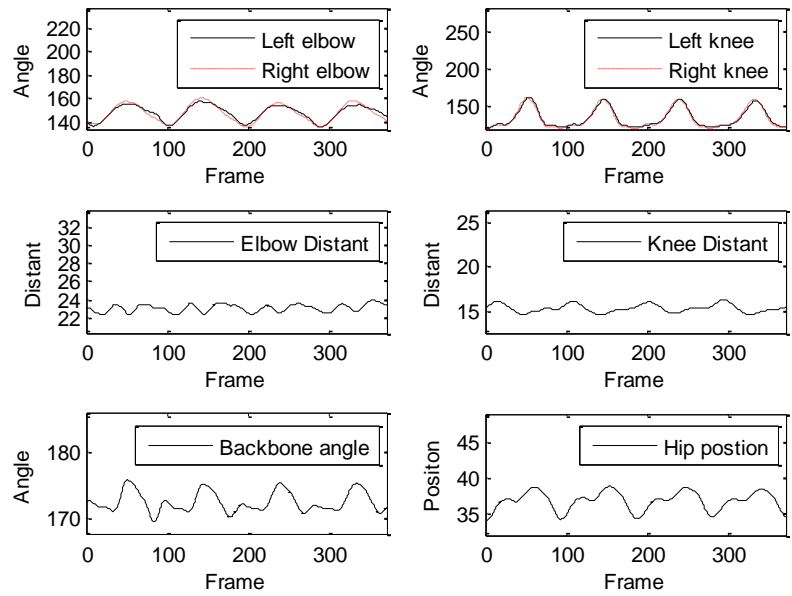

Fig. 11. Feature values of Thoroughbred at a trot

\section{Thoroughbred at a Canter}

Figure 12 shows that repeating 300 frames were extracted out of approximately 10000 frames to demonstrate 4 cycles ( 3 seconds). As shown in the Figure below, the elbow and knee angles remain at the range of about 135 160 degrees and about 120 140 degrees, respectively. The elbow and knee distances stay at the range of $22 \sim 25 \mathrm{~cm}$ and $13 \sim 19 \mathrm{~cm}$, respectively. The backbone angle remains at the range of 170 177 degrees. The Hip positions moves within the range of $31 \sim 38 \mathrm{~cm}$. Figure 12 shows the average feature values of 15 data.
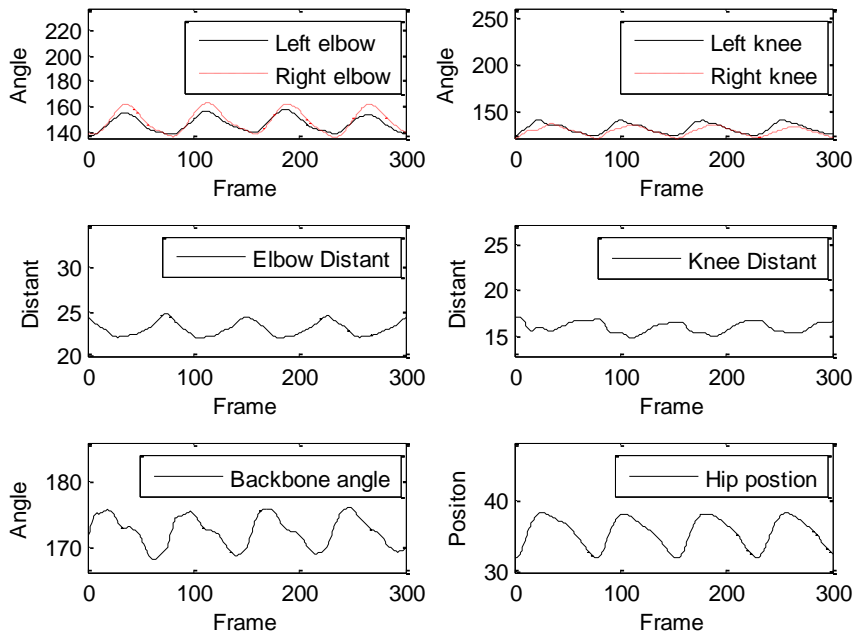

Fig. 12. Feature values of Thoroughbred at a canter

Figure 13 below illustrates the numerical comparison of feature values of two horses at a rising trot. A visible difference is revealed in the elbow angles. A similar difference is noticed in knee angles as shown in the elbow angles. The reason why no significant difference was made in the backbone angles and in the hip positions is because the horse- rider should keep her backbone at its perpendicularity and her hip at the same position whatever type of a horse she is riding on.

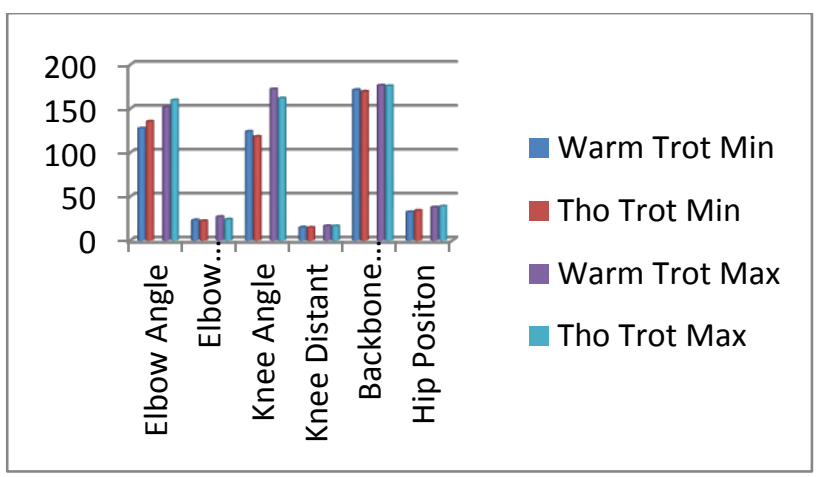

Fig. 13. Comparison of maximum and minimum feature values at a trot

Figure 14 below illustrates the numerical comparison of maximum and minimum feature values of two horses (Warmblood and Thoroughbred) at a canter. A visible difference is revealed in the elbow angles. A similar difference is noticed in knee angles as shown in the elbow angles. As described above, no significant difference was made in the backbone angles and in the hip positions because the horse-rider should keep her backbone at its perpendicularity and her hip at the same position regardless of the type of horses.

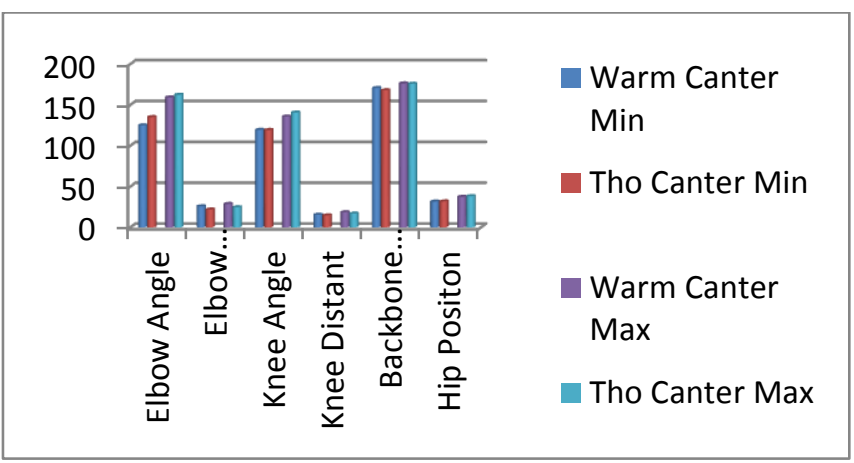

Fig. 14. Comparison of maximum and minimum feature values at a canter

\section{CONCLUSIONS}

This paper suggested a method of using a motion database of a professional horse-rider wearing a suit constructed with wireless networks consisting of 16 inertial sensors and then extracting the respective motion features (elbow angle, knee angle, backbone angle, hip position, knee-elbow distance) through various calculation methods such as Euclidean distance, the second cosine, maximum and minimum values, depending on horse types (Warm-blood and Thoroughbred) and footpace types (trot and canter). MVN studio software was used to make a comparative analysis on the horse-rider's motion features depending on the footpace types. As a result, a significant difference was noticed in the motion feature values obtained depending on different horse and footpace types. Therefore, in order to effectively make real-time coaching sessions for different horse-riding footpace types, it is necessary to construct a motion feature database in relation to footpace types and accordingly make a suitable analysis and coach on horse-riding motions. 


\section{REFERENCES}

[1] The MTx system, xSens, http://www.xsens.com/

[2] Horse riding, http://terms.naver.com/entry.nhn?docId=384601\&cid= 689 \& category $\mathrm{Id}=1458$

[3] How to Horse riding, http://ko.wikipedia.org/wiki\%EX\%8A\%B9\%EB $\%$ A7\%88

[4] H. J. Luinge, and P. H. Veltink, "Measuring Orientation of Human Body Segments Using Miniature Gyroscopes and Accelerometers," Medical \& Biological Engineering \& Computing, vol. 43, no. 2, pp. 273-282, 2005.

[5] H. Zhou, T. Stone, H. Hu, and N. Harris, "Use of Multiple Wearable Inertial Sensors in Upper Limb Motion Tracking," Medical Engineering \& Physics, vol. 30, no. 1, pp. 123-133, 2008.

[6] J. Lee, and I. Ha, "Sensor fusion and calibration for motion captures using accelerometers," Proc. IEEE International Conference on Robotics and Automation, 1999, pp. 1954-1959.

[7] R. Zhu, and Z. Zhou, "A Real-Time Articulated Human Motion Tracking Using Tri-Axis Inertial/Magnetic Sensors Package," IEEE Transactions on Neural Systems and Rehabilitation Engineering, vol. 12, no. 2, pp. 295-302, 2004.

[8] L. Cheng, S. Hailes, "An Experimental Study on a Motion Sensing System for Sports Training," short paper in the Proceedings of the $5^{\text {th }}$ European Conference on Wireless Sensor Networks (EWSN), Bologna, Italy, Feb 2008.

[9] S. Venkatraman, J. Long, K. Pister, J. Carmena, "Wireless Inertial Sensors for Monitoring Animal Behaviour," in Proceedings of the IEEE Engineering in Medicine and Biology Society (EMBS), Lyon, France, Aug 2007, pp. 378-381.

[10] H. Ghasemzadeh, V. Loseu, E. Guenterberg, and R. Jafari, "Sport training using body sensor networks: a statistical approach to measure wrist rotation for golf swing," in BodyNets '09: Proceedings of the Fourth International Conference on Body Area Networks, 2009, pp. 1-8.

[11] K. Kevin, S. W. Yoon, N.C. Perkins, "Wireless mems inertial sensor system for golf swing dynamics," Sensors and Actuators A: Physical, vol. 141 , no. 2 , pp. $619-630,2008$.

[12] Mariani B, Hoskovec C, Rochat S, Bula C, Penders J, Aminian K. "3D gait assessment in young and elderly subjects using foot-worn inertial sensors," J Biomech, 2010;43(15), pp.2999-3006.

[13] J. Yujin, K. Donghoon, K. Jinwook, "Upper Body Motion Tracking With Inertial Sensors," proc. IEEE International Conference on Robotics and Biomimetics, 2010, pp. 1746-1751.
[14] Song Lijun, Qin Yongyuan, "A Practical Calibration Method on MEMS Gyroscope". Piezoelectrics \& Acoustooptics, Vol.32, No.3, 2010, pp.372-374

[15] R. Wei, Z. Tao, Z haiyun, W. Leigang, "A Research on Calibration of Low-Precision MEMS Inertial Sensors," IEEE Transactions on Control and Decision Conference, 2013, pp. 3243-3247.

[16] N. Cao, S. Young, K. Dang, "3D Dynamics Analysis of a Golf Full Swing by Fusing Inertial Sensor and Vision data," International Conference on Control, Automation and Systems, 2013, pp. 1300-1303.

[17] P. G. Jung, G. Lim, K. Kong, “A Mobile Motion Capture System Based on Inertial Sensors and Smart Shoes," IEEE Transactions Robotics and Automation, 2013, pp. 692-697.

[18] J. C. P. Chan, H. Leung, J. K. T. Tang, and T. Komura, "A virtual reality dance training system using motion capture technology," IEEE Transactions on Learning Technologies, vol. 4, no. 2, pp. 187.195, 2011.

[19] [19] I. Frosio, F. Pedersini, and N. A. Borghese, "Autocalibration of MEMS accelerometers," IEEE Transactions on Instrumentation and Measurement, vol. 58, no. 6, pp. 2034-2041, 2009.

[20] Wireless sensor network, http://en.wikipedia.org/wiki/ Wireless_sensor_ network

\section{AUTHORS PROFILE}

Jae-Neung Lee received the B.Sc. from Chosun University, Gwangju, Korea, in 2013. He is currently pursuing a candidate for the M.Sc. His research interests include human-robot interaction, computational intelligence, and pattern recognition.

Keun-Chang Kwak received the B.Sc., M.Sc., and Ph.D. degrees from Chungbuk National University, Cheongju, Korea, in 1996, 1998, and 2002, respectively. During 2003-2005, he was a Postdoctoral Fellow with the Department of Electrical and Computer Engineering, University of Alberta, Edmonton, AB, Canada. From 2005 to 2007, he was a Senior Researcher with the Human-Robot Interaction Team, Intelligent Robot Division, Electronics and Telecommunications Research Institute, Daejeon, Korea. He is currently the Associative Professor with the Department of Control and Instrumentation, Engineering, Chosun University, Gwangju, Korea. His research interests include human-robot interaction, computational intelligence, biometrics, and pattern recognition. Dr. Kwak is a member of IEEE, IEICE, KFIS, KRS, ICROS, KIPS, and IEEK. 